\title{
Knowledge and Use of Ethnomedical Treatments for Asthma Among Puerto Ricans in an Urban Community
}

Luis E. Zayas, $P b D^{1}$

Angela M. Wisniewski, PharmD $D^{2,3}$

Renee B. Cadzow, $P b D^{2}$

Laurene M. Tumiel-Berbalter, $P b D^{2}$

'School of Social Work, College of Public Programs, Arizona State University, Phoenix, Arizona

${ }^{2}$ Department of Family Medicine, School of Medicine and Biomedical Sciences, University of Buffalo, Buffalo, New York

${ }^{3}$ Department of Pharmacy Practice, School of Pharmacy and Pharmaceutical Sciences, University at Buffalo, Buffalo, New York
Conflicts of interest: authors report none.

\section{CORRESPONDING AUTHOR}

Luis E. Zayas, PhD

School of Social Work

Arizona State University

411 North Central Avenue, Suite 800

Phoenix, AZ 85004

lezayas@asu.edu

\begin{abstract}
PURPOSE Puerto Ricans have higher lifetime and current asthma prevalence than other racial and ethnic groups in the United States. A great many Hispanics use ethnomedical therapies for asthma. This study elicited participant knowledge of ethnomedical therapies, developed a typology of the therapies, and considered whether some types are used or deemed efficacious based, in part, on information source.
\end{abstract}

METHODS Eligible participants were randomly selected from the medical records of an inner-city primary care clinic serving a predominantly Hispanic community in Buffalo, New York. Thirty adult Puerto Ricans who had asthma or were caregivers of children with asthma were interviewed in person using a semistructured instrument. Qualitative data analysis followed a content-driven immersion-crystallization approach. Outcome measures were ethnomedical treatments for asthma known to participants, whether these treatments were used or perceived effective, and the participant's information source about the treatment.

RESULTS Participants identified 75 ethnomedical treatments for asthma. Behavioral strategies were significantly more likely to be used or perceived effective compared with ingested and topical remedies $(P<.001)$. Among information sources for ingested and topical remedies, those recommended by community members were significantly less likely to be used or perceived effective $(P<.001)$ compared with other sources.

CONCLUSIONS This sample of Puerto Ricans with a regular source of medical care was significantly more likely to use or perceive as effective behavioral strategies compared with ingested and topical remedies. Allopathic clinicians should ask Puerto Rican patients about their use of ethnomedical therapies for asthma to better understand their health beliefs and to integrate ethnomedical therapies with allopathic medicine.

Ann Fam Med 2011;9:50-56. doi:10.1370/afm.1200.

\section{INTRODUCTION}

n general, Hispanics have worse access to care, and the health care they receive is of poorer quality than that received by non-Hispanic whites. ${ }^{.}$Compared with other Hispanic subgroups (eg, MexicanAmericans, Cubans), Puerto Ricans have poorer self-assessed health status, more activity limitations, and more medical conditions. ${ }^{2,3}$ This disparity exists despite their eligibility for public health care programs as US citizens. Lifetime and current asthma prevalence are higher in Puerto Rico than any other state or territory of the United States. ${ }^{4-6}$ Among Hispanics in the United States, Puerto Rican children and adults have higher lifetime asthma prevalence, ${ }^{6-13}$ current asthma prevalence, ${ }^{6-8,13-16}$ and age-adjusted mortality ${ }^{17}$ than do other racial (eg, non-Hispanic white, non-Hispanic black) and ethnic groups, including Hispanic subgroups (eg, Mexican- 
Americans, Dominicans, Cubans). One study found Puerto Ricans have higher current asthma prevalence than Dominicans and other Hispanic subgroups, even when they have the same living environment and a similar socioeconomic status. ${ }^{18}$ The reasons for this disparity are not known. In Buffalo, New York, where this study was conducted, Hispanics, most of whom are of Puerto Rican descent, are more likely to have asthma than African Americans, and asthma prevalence among Hispanic children is twice that of white children. ${ }^{19,20}$

Cornerstones of asthma management include lifestyle adjustments and conventional pharmacologic medications. Studies have found as many as $72 \%$ to $89 \%$ of Hispanics use or are aware of ethnomedical, complementary and alternative medicine (CAM), and home or folk remedies for asthma. ${ }^{21-28}$ In light of the high prevalence of asthma among Puerto Ricans and the great number of Hispanics who use ethnomedical therapies for asthma, it is important to understand which ethnomedical treatments Puerto Ricans use. Many studies of ethnomedical use in general ${ }^{29,30}$ and asthma specifically ${ }^{21,22,25}$ have grouped Hispanics together into 1 category for data presentation and analysis, focused on other Hispanic subgroups (eg, Dominican), ${ }^{24}$ or included only small numbers of Puerto Ricans. ${ }^{23}$ Few studies have focused on mainland Puerto Ricans ${ }^{26-28}$ and examined the influence of ethnomedical treatment type $\mathrm{e}^{26-28}$ and information source ${ }^{25}$ on use or perceived efficacy. In the context of this study, ethnomedical treatments are nonallopathic treatments that are part of a system of healing practices, beliefs, and social relations of an ethnic group, derived mostly from the popular (lay, nonprofessional, nonspecialist) and folk (nonprofessional, specialist) health care sectors. ${ }^{31}$

This qualitative study addresses the following 3 research questions: (1) What types of ethnomedical treatments for asthma are Puerto Ricans with a regular source of care aware of? (2) Which specific ethnomedical treatments do they use (or consider effective)? (3) Does the information source for the ethnomedical treatment influence whether it is used? The answers to these questions could help health care clinicians to assess the use of ethnomedical treatments for asthma by their Puerto Rican patients and to integrate these treatments with allopathic therapy.

\section{METHODS}

We used a qualitative phenomenological design with individual in-depth interviews useful for exploring the lived experiences of participants with a particular phenomenon (eg, asthma). ${ }^{32}$ Using a purposeful sampling strategy, which randomly selects from a developed sampling frame to enhance credibility without claim- ing to be representative, ${ }^{33}$ we selected patients from an inner-city, primary care clinic in Buffalo, New York, that serves the Hispanic community. The clinic averages 1,800 office visits each month, approximately twothirds of which are by Hispanic patients. Patients who had an asthma-related office visit in the prior 2 years were identified from the medical records and stratified by the number of such visits ( 0 to 1,2 to 4 , and 5 or more) as a proxy for asthma severity, as severity was infrequently documented. An equal number from each stratum were randomly selected and invited to participate in the study. For patients younger than 18 years, the child's parent or guardian was interviewed. A bilingual Puerto Rican researcher (L.E.Z.), trained in qualitative research, conducted the interviews in Spanish or English at the participant's home or at the clinic, depending on their preference. The University at Buffalo Institutional Review Board approved the study, and all participants provided written, informed consent.

Of 36 adults who consented to participate, 6 were excluded from subsequent analysis. One interview was incomplete, 2 were recorded on a damaged audiotape, and 3 involved participants who were not Puerto Rican. Hence, a total of 30 Puerto Rican patient or caregiver interviews were analyzed; 23 (77\%) were conducted at the patient's home and 7 (23\%) at the clinic. Twentyfive $(83 \%)$ interviews were conducted in Spanish.

The interviews lasted 60 to 90 minutes and were audio-recorded, transcribed, and translated into English for analysis and reporting. All respondents completed a brief demographic exit questionnaire. Depth interviewing was a practical strategy for this study because it is designed to elicit narrative from the participant's point of view, allowing the interviewer to probe responses indepth to obtain sufficient description and context, and this type of interviewing is particularly useful for eliciting free listings of items of inquiry. ${ }^{34}$

This preliminary study, part of an initiative to identify asthma management and coping strategies used by Hispanics, was based primarily on responses to 2 open-ended questions and 1 key probe pertaining to ethnomedical asthma treatments: (1) What do you do to treat or take care of your (or your child's) asthma? (2) What alternative ways of treating or dealing with asthma, such as home remedies or other forms of treatment do you know of? And, if any, What do you think about them? Data from the entire interview — which included responses to 12 other open-ended questions-were also reviewed, and any information pertinent to ethnomedical therapies for asthma was also considered in the analysis.

Transcriptions were entered into NVivo 2.0 (QSR International Pty Ltd, Doncaster, Victoria, Australia), ${ }_{1}^{35}$ qualitative data management software for analysis. Two 
medical anthropologists (L.E.Z., R.B.C.) experienced in phenomenological analysis reviewed the transcripts according to a content-driven, immersion-crystallization approach. This approach consists of an iterative process of data review and interpretation in which the analysts delve deep into or immerse themselves in the data, experience and reflect upon it, and develop or crystallize insights that can be synthesized to establish patterns and connections. ${ }^{36}$ First, the analysts independently reviewed the participants' responses to the study questions to identify references to nonpharmacologic asthma therapies, opinions about efficacy, information sources, and other descriptive or contextual information. Then they catalogued all the therapies through consensus. Last, the analysts separately reviewed each transcript in its entirety in search of additional supporting or conflicting evidence and updated the catalog accordingly. This search for missing and contradictory evidence in the transcripts serves to enhance trustworthiness. ${ }^{37}$

For each ethnomedical treatment, the analysts recorded the number of participants who identified that treatment, whether the therapy was used (or deemed efficacious), and the source of information. These quantitative data were entered into an Excel spreadsheet and analyzed using SPSS, version 16.0 (SPSS Inc, Chicago, Illinois) by the study's epidemiologist (L.T.B.) and pharmacologist researcher (A.M.W.). $\chi^{2}$ Analysis was used to compare use or perceived efficacy of ethnomedical treatment modalities (ingested and topical remedies vs behavioral strategies) and the effect of information source on use or perceived efficacy. A $P$ value $<.05$ was considered statistically significant.

\section{RESULTS}

The characteristics of the interviewees, all of whom self-identified as Puerto Rican, are provided in Table 1.

Among a total of 161 treatments mentioned, participants identified 75 ethnomedical therapies for asthma. All but 1 respondent reported knowing of at least 1 treatment, and 1 participant reported 15 different treatments. Ethnomedical therapies were classified into 2 general categories: ingested and topical remedies, and behavioral strategies (Table 2). Several of these ethnomedical treatments have previously been reported in the literature, whereas others are new. Below follows a brief narrative of the more common therapies and a description correlating use or perceived efficacy with the information source.

\section{Ingested and Topical Remedies}

The most common subcategories of ingested and topical remedies involved over-the-counter products and herbal concoctions. Vicks VapoRub was the single most mentioned therapy, with several mothers indicating they applied Vicks to their child's back and chest to ease breathing. Herbal tea, rubbing alcohol, and honey were the other 3 most frequently mentioned ingested and topical remedies. For example, the mother of a child with asthma mentioned:

Many times, I put Vicks on his back [and] chest.... Also, it helps a little [if] I wash him in [rubbing] alcohol from head to toe... because he gets hot, all red in the face.... The alcohol is cold.... I leave it in the refrigerator. I wash him in alcohol...so that he can get cool [and] calm down, to help a little bit if the medicine does not help quickly....

Several respondents also noted the use of natural products, such as honey and Aloe vera, as expectorants and decongestants to loosen phlegm. One mother, for example, cited "...giving him honey so he could cough out that junk...," and another respondent observed that "...[Aloe vera] helps me with the phlegm, doesn't let it get so much...." Another respondent mentioned, "I eat garlic every night before going to bed, raw, and it seems to have helped a lot...." Citrus fruits, such as

\begin{tabular}{|c|c|}
\hline Characteristic & Value \\
\hline \multicolumn{2}{|l|}{ Sex, No, (\%) } \\
\hline Male & $5(17)$ \\
\hline Female & $25(83)$ \\
\hline Age, mean (range) y & $38.4(20-84)$ \\
\hline \multicolumn{2}{|l|}{ Interviewee, No. (\%) } \\
\hline Parent of child $(\leq 17 \mathrm{y})$ with asthma & $13(43)$ \\
\hline Adult ( $\geq 18$ y) with asthma & $17(57)$ \\
\hline \multicolumn{2}{|l|}{ Education, No. (\%) } \\
\hline$\leq 8$ th grade & $7(23)$ \\
\hline Some high school & $8(27)$ \\
\hline High school graduate or GED & $6(20)$ \\
\hline$\geq$ Some college & $9(30)$ \\
\hline \multicolumn{2}{|l|}{ Employment status, No, (\%) } \\
\hline Full-time & $10(33)$ \\
\hline Part-time & $3(10)$ \\
\hline Unemployed & $10(33)$ \\
\hline Not working due to disability & $7(23)$ \\
\hline \multicolumn{2}{|l|}{ Annual household income, No, (\%) } \\
\hline$<\$ 5,000$ & $6(20)$ \\
\hline$\$ 5,000-\$ 9,999$ & $15(50)$ \\
\hline$\$ 10,000-\$ 20,000$ & $3(10)$ \\
\hline$\geq \$ 20,000$ & $6(20)$ \\
\hline \multicolumn{2}{|l|}{ Marital status, No, (\%) } \\
\hline Married & $16(53)$ \\
\hline Separated or divorced & $5(17)$ \\
\hline Widowed & $2(7)$ \\
\hline Never married & $7(23)$ \\
\hline Household size, mean (range), No. & $4.3(1-10)$ \\
\hline Household members with asthma, mean (range), No. & $1.5(1-6)$ \\
\hline
\end{tabular}


orange, lemon, and grapefruit, were also mentioned for treating asthma symptoms: "I was next to a place where people were smoking... already I felt the wheez-

\section{Table 2. Types of Ethnomedical Asthma Treatments Cited by Respondents}

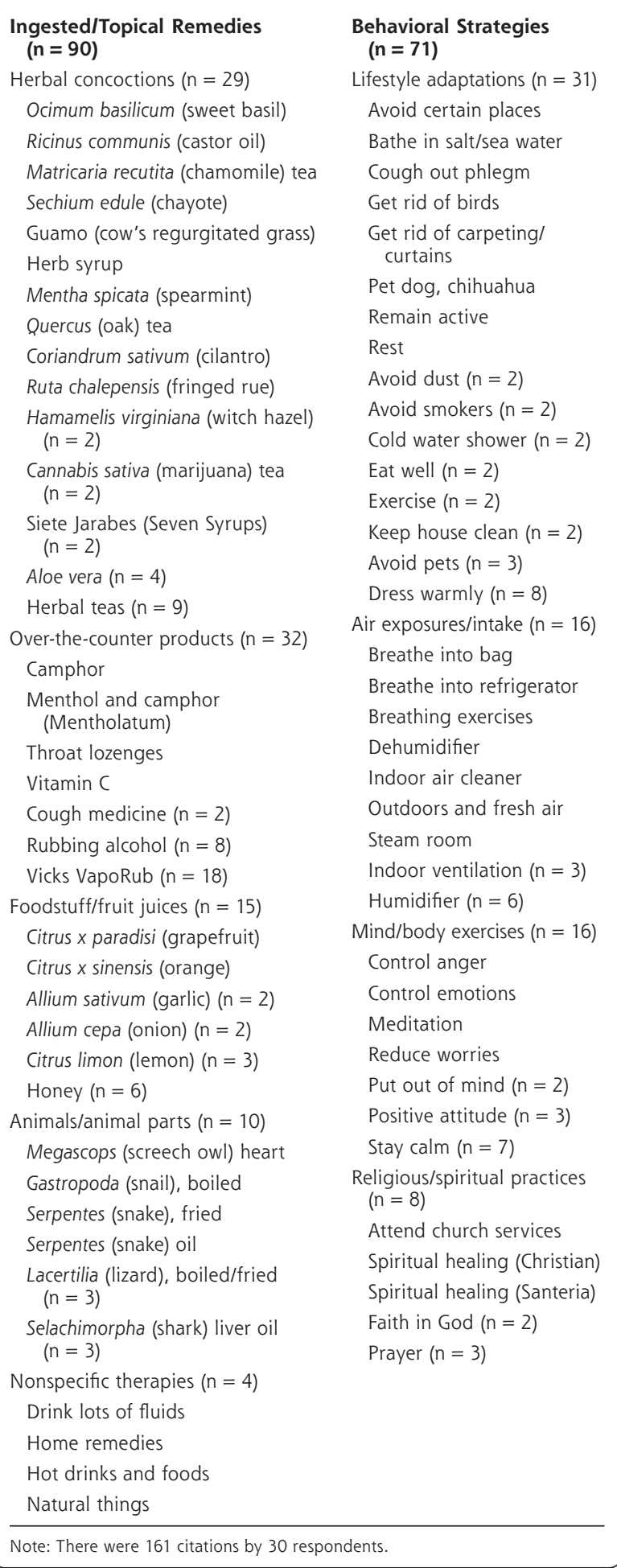

ing symptoms.... I ate have been able to incorporate all your requested changes. Attached is a revised first proof a pair of oranges and a sour grapefruit... and that's it."

\section{Behavioral Strategies}

Lifestyle adaptations were the most common subcategory of behavioral strategies for dealing with asthma, whereas dress warm, stay calm, and humidifier were the 3 most commonly mentioned behavioral strategies. For example, a parent recounted,

..make sure she has socks at all times and T-shirt underneath her shirt. When she goes outside, make sure she always has a hat...[and that] no draft would go into her coat.

Another respondent mentioned, "...not getting scared, staying calm...I used to start crying and get desperate when I got asthma, [and] I would get worse...." Another one noted, "my asthma is more emotional than anything else. If I get mad, I try to control myself and don't let it get to me...." Several respondents also cited strategies to improve air quality:

I asked [my doctor] about a vaporizer...especially for [the child's] room... he said there's nothing wrong with that [but] not to put Vicks liquid in there and make sure it's only with water.

Religious and spiritual practices were mentioned too, as several participants indicated that prayer, attendance at mass, or faith in God could help alleviate asthma symptoms. One respondent, for example, believed that "the most important thing is to have faith in God... if you also have strong faith and pray... Jesus will hear you."

\section{Utilization and Efficacy}

Participants were probed about their opinions on identified ethnomedical therapies, specifically whether they used them, their efficacy, and how they learned about them. Table 3 displays use or perceived efficacy by type of ethnomedical treatment (ingested and topical

\section{Table 3. Type and Use of Ethnomedical Asthma} Treatments

\begin{tabular}{lcc}
\hline $\begin{array}{l}\text { Type of } \\
\text { Treatment }\end{array}$ & $\begin{array}{c}\text { Treatment Used } \\
\text { (or Deemed } \\
\text { Effective) No. (\%) }\end{array}$ & $\begin{array}{c}\text { Treatment Not } \\
\text { Used (or Deemed } \\
\text { Ineffective) No. (\%) }\end{array}$ \\
\hline $\begin{array}{l}\text { Ingested } \\
\text { and topical } \\
\text { remedies } \\
\text { Behavioral } \\
\text { strategies }\end{array}$ & $28(31)$ & $62(69)$ \\
\hline $\begin{array}{l}\text { Note: There were } 161 \text { citations by } 30 \text { respondents. P <.001 for the compari- } \\
\text { son use of Ingested and topical remedies vs behavioral strategies. }\end{array}$ \\
\hline
\end{tabular}




\begin{tabular}{llccc} 
Table 4. Stratified Analysis of Type and Use of Ethnomedical & $\begin{array}{c}\text { Asthma Treatments by Information Source } \\
\text { Ingested/Topical Remedies } \\
\mathbf{N}=90\end{array}$ & $\begin{array}{c}\text { Behavioral Strategies } \\
\mathbf{N}=\mathbf{7 1}\end{array}$ \\
\cline { 2 - 5 } & $\begin{array}{c}\text { Treatment Used } \\
\text { (or Deemed Effective) } \\
\text { No. (\%) }\end{array}$ & $\begin{array}{c}\text { Treatment Not Used } \\
\text { (or Deemed Ineffective) } \\
\text { No. (\%) }\end{array}$ & $\begin{array}{c}\text { Treatment Used } \\
\text { (or deemed Effective) } \\
\text { No. (\%) }\end{array}$ & $\begin{array}{c}\text { Treatment Not Used } \\
\text { (or Deemed Ineffective) } \\
\text { No. (\%) }\end{array}$ \\
\hline Self & $3(11)$ & $0(0)$ & $6(9)$ & $0(0)$ \\
Relative & $10(36)$ & $15(24)$ & $16(25)$ & $3(50)$ \\
Community & $0(0)$ & $40(65)$ & $7(11)$ & $2(33)$ \\
Clinician & $0(0)$ & $0(0)$ & $6(9)$ & $0(0)$ \\
Not specified & $15(54)$ & $7(11)$ & $30(46)$ & $1(17)$
\end{tabular}

Note: There were 161 citations by 30 respondents. $P<.001$ for the comparison use of ingested/topical remedies by information source. The comparison for use of behavioral strategies by information source was not statistically significantly different.

remedies vs behavioral strategies). Significantly more participants reported current use or perceived efficacy of behavioral strategies compared with current use or perceived efficacy of ingested and topical remedies (92\% vs $31 \%$, respectively, $P<.001$ ).

Among specified information sources, participants learned about ethnomedical asthma therapies mostly from the community (49 mentions), which usually referred to people in their neighborhood, and relatives (44 mentions) (Table 4). Although the community was the primary source of information on ingested and topical remedies (40 of 90,44\%), these remedies were significantly less likely to be currently used or judged effective compared with remedies from other information sources $(P<.001)$. For example, 1 participant recounted that "in Puerto Rico they would say 'drink this tea, that will cure you', but never did...." Another one stated, "People told me... shark oil is very good for [asthma]...but it never did anything for me." Although the information source was not found to determine whether behavioral strategies were used or deemed effective, unspecified sources (30 of $71,42 \%$ ) were the most common source of information for behavioral strategies, followed by relatives (19 of $71,27 \%$ ). As another respondent mentioned, "My mother-in-law told us that we couldn't have birds because their waste and their feathers do harm...."

All (15 of 15) ethnomedical asthma treatments that were self-identified, recommended by a clinician, or for which a clinician's advice was sought were used or judged to be effective. Some participants, for example, indicated that they preferred to consult with their clinician first: "I do not criticize them. It might be true. But if a doctor doesn't give it to me...." One parent mentioned,

[People] told me to use witch hazel and Aloe vera and something else, you mix it and give it to [child] and the asthma will go away forever! [Laughs] ... if I'm gonna use something else I got to talk to the doctor....
Although relatives were more persuasive than community members, the final word for many participants came from their clinician: "My sister-in-law says you cannot give Vicks to children with asthma. You know, there are many Hispanic beliefs, so I'd like to [verify] with the doctor..."

\section{DISCUSSION}

This sample of Puerto Rican participants documented ingested and topical remedies and behavioral strategies for asthma, but they were significantly more likely to use behavioral strategies or perceive them as effective. Although relatives and the community were frequent sources of ethnomedical treatment information, participants themselves and their health care clinicians were the most influential information sources regarding use of or confidence in a particular treatment. In fact, all treatments for which the participant or a health care clinician was the information source were used or perceived as effective. None of the ingested or topical remedies recommended by the community was currently used or deemed effective.

These findings are consistent with previous studies. A study involving caregivers of mainland Puerto Rican children with asthma found that the most commonly used ethnomedical strategies to prevent an asthma attack and to treat an exacerbation were behavioral strategies. ${ }^{26}$ In a study that interviewed caregivers of children with asthma, participants residing in Puerto Rico (both Puerto Rican and Dominican) were more likely to view biologically based practices as effective for treating asthma, whereas participants from Rhode Island (both Puerto Rican and Dominican) were more likely to favor mind-body practices. ${ }^{27}$

Other studies have also found that the information source may have an effect on use of ethnomedical or CAM therapies. An analysis of National Health Interview Survey data found that approximately 14\% 
of Hispanics had used a CAM therapy at the suggestion of an allopathic medical professional. ${ }^{29}$ A study of CAM use among asthmatic adolescents found that participants were significantly more likely to use CAM again if they had a family member who used CAM and if they perceived CAM to be effective. ${ }^{25}$ Another study determined that perceived efficacy of biomedical and ethnomedical therapies may depend on whether the condition being treated is considered a folk illness. ${ }^{30}$

Studies have found that Hispanic users of CAM $(69 \%),{ }^{29}$ including CAM for asthma (46\% to $\left.82 \%\right),{ }^{22,25}$ often do not disclose use of these therapies to their health care clinician, which could pose a problem if a CAM therapy contained a potentially harmful substance. Although most treatments mentioned in this study are relatively harmless (eg, mind-body exercises, religious and spiritual practices) or even recommended (eg, avoiding pets and smoke), there are some with the potential to cause harm (eg, application of rubbing alcohol, particularly on young children). Additionally, for many of the ingested and topical remedies, particularly among the herbal concoctions, potential interactions with prescribed allopathic therapies are not well characterized.

Conceptually, at least 2 explanatory models of illness may support use of these ethnomedical therapies. One commonly identified in the literature on Puerto Ricans is the concept of balance (physical, emotional, social, or spiritual) based upon the humoral theory whereby illness (eg, asthma) results from an imbalance of hot and cold. ${ }^{26,28,38,39}$ There were several mentions of behavioral strategies involving staying calm or warm, and numerous mentions of ingested and topical remedies that were hot or cold. This finding suggests that it is important for many to restore emotional and physical balance to alleviate asthma symptoms. In a study that compared variations in asthma beliefs and practices among mainland Puerto Ricans, MexicanAmericans, Mexicans, and Guatemalans, researchers found that these groups shared a belief system regarding asthma, particularly the concept of balance and humoral causes, though systematic variation existed between groups in asthma treatments. ${ }^{28}$ Another possible framework not discussed in this literature is the plumbing model of the body, ${ }^{39}$ whereby illness results when the flow of various substances (eg, blood, air, food, urine) is interrupted in the body. Many examples of ingested and topical and behavioral therapies cited in this study were aimed at unclogging air passages and improving air intake and flow.

Study findings are consistent with those of previous studies indicating that mainland Puerto Ricans with asthma (or their caregivers) are more likely to use behavioral strategies. In addition, we are not aware of any previous study in which use or perceived efficacy of ingested and topical remedies and behavioral strategies were evaluated in relation to their information source.

This study has several limitations. Findings were based on qualitative interviews with 30 Puerto Rican participants who were established patients at an urban health care center. According to Lincoln and Guba, ${ }^{40}$ for qualitative findings to be transferable (the corresponding qualitative term for generalizable), the study contexts must be similar. As such, results should not be transferred to those who do not have a regular source of medical care. These results may not be transferable to Puerto Ricans with asthma living in different social contexts or geographic areas, nor may our findings be reflective of the practices of other Hispanics subgroups with asthma. In addition, the level of acculturation of the respondents was not assessed to discern potential differences in treatment knowledge and use. Finally, self-identified treatments could have been learned from an unrecognized source.

This comprehensive inventory of ethnomedical therapies for asthma, although not exhaustive, illustrates the vast diversity of ethnomedical therapeutic strategies used by Puerto Ricans in the United States. How these therapies fit within the broad categories of ingested and topical remedies and behavioral strategies and their subcategories may serve as a useful framework for health care clinicians in determining their advice to patients regarding use of ethnomedical therapies. The lower rate of use of ingested and topical remedies in this sample is interesting and warrants further research using a larger sample from different geographic areas and controlling for access to medical care. Greater use or perceived efficacy of behavioral strategies, as opposed to ingested and topical remedies, lends itself to integration of these practices with conventional allopathic medicine. Pachter et al noted that "some home-based modalities fit well within standard biomedical practices." ${ }^{26}$ Knowledge of ethnomedical and CAM treatments and understanding a patient's health belief model may give a health care clinician insight into how a patient conceptualizes illness, hence the importance of probing about use of ethnomedical treatments for asthma regardless of the ethnic group. Such background information may allow the health care clinician and patient to determine a course of treatment consistent with the patient's beliefs.

To read or post commentaries in response to this article, see it online at http://www.annfammed.org/cgi/content/full/9/1/50.

Key words: Asthma; ethnomedicine; folk medicine; complementary therapies; alternative medicine; qualitative methods

Submitted March 13, 2010; submitted, revised, July 1, 2010; accepted July 22, 2010. 
Acknowledgements: The authors would like to thank Andy Danzo for his critical review of the manuscript.

\section{References}

1. US Department of Health and Human Services. National Healthcare Disparities Report: 2009. Rockville, MD: Agency for Healthcare Research and Quality; 2010.

2. Hajat A, Lucas JB, Kington R. Health outcomes among Hispanic subgroups: United States, 1992-1995. Adv Data. 2000;25(310):1-15.

3. Zsembik BA, Fennell D. Ethnic variation in health and the determinants of health among Latinos. Soc Sci Med. 2005;61(1):53-63.

4. Pérez-Perdomo R, Pérez-Cardona C, Disdier-Flores O, Cintrón Y. Prevalence and correlates of asthma in the Puerto Rican population: Behavioral Risk Factor Surveillance System, 2000. J Asthma. 2003;40(5):465-474.

5. Centers for Disease Control and Prevention (CDC). Asthma prevalence and control characteristics by race/ethnicity-United States, 2002. MMWR Morb Mortal W/kly Rep. 2004;53(7):145-148.

6. Lara M, Akinbami L, Flores G, Morgenstern H. Heterogeneity of childhood asthma among Hispanic children: Puerto Rican children bear a disproportionate burden. Pediatrics. 2006;117(1):43-53.

7. Rose D, Mannino DM, Leaderer BP. Asthma prevalence among US adults, 1998-2000: role of Puerto Rican ethnicity and behavioral and geographic factors. Am J Public Health. 2006;96(5):880-888.

8. Carter-Pokras OD, Gergen PJ. Reported asthma among Puerto Rican, Mexican-American, and Cuban children, 1982 through 1984. Am J Public Health. 1993;83(4):580-582.

9. Quinn K, Shalowitz MU, Berry CA, Mijanovich T, Wolf RL. Racia and ethnic disparities in diagnosed and possible undiagnosed asthma among public-school children in Chicago. Am J Public Health 2006;96(9):1599-1603.

10. Beckett WS, Belanger K, Gent JF, Holford TR, Leaderer BP. Asthma among Puerto Rican Hispanics: a multi-ethnic comparison study of risk factors. Am J Respir Crit Care Med. 1996;154(4 Pt 1):894-899.

11. Davis AM, Kreutzer R, Lipsett M, King G, Shaikh N. Asthma prevalence in Hispanic and Asian American ethnic subgroups: Results from the California Healthy Kids survey. Pediatrics. 2006;118(2):e363-e370.

12. Freeman NC, Schneider D, McGarvey P. Household exposure factors, asthma, and school absenteeism in a predominantly Hispanic community. J Expo Anal Environ Epidemiol. 2003;13(3):169-176.

13. Akinbami LJ, Rhodes JC, Lara M. Racial and ethnic differences in asthma diagnosis among children who wheeze. Pediatrics. 2005; 115(5):1254-1260.

14. Dumanovsky T, Matte TD. Variation in adult asthma prevalence in Hispanic subpopulations in New York City. J Asthma. 2007;44(4): 297-303.

15. Centers for Disease Control and Prevention. National surveillance for asthma - United States, 1980-2004. MMWR Morb Mortal W/kly Rep. 2007;56(1):1-60.

16. Claudio L, Stingone JA, Godbold J. Prevalence of childhood asthma in urban communities: the impact of ethnicity and income. Ann Epidemiol. 2006;16(5):332-340.

17. Homa DM, Mannino DM, Lara M. Asthma mortality in U.S. Hispanics of Mexican, Puerto Rican, and Cuban heritage, 1990-1995. Am J Respir Crit Care Med. 2000;161(2 Pt 1):504-509.

18. Ledogar RJ, Penchaszadeh A, Garden CC, Iglesias Garden. Asthma and Latino cultures: different prevalence reported among groups sharing the same environment. Am J Public Health. 2000;90(6):929-935.

19. Lwebuga-Mukasa JS, Dunn-Georgiou E. The prevalence of asthma in children of elementary school age in western New York. J Urban Health. 2000;77(4):745-761.
20. Lwebuga-Mukasa JS, Wojcik R, Dunn-Georgiou E, Johnson C. Home environmental factors associated with asthma prevalence in two Buffalo inner-city neighborhoods. J Health Care Poor Underserved. 2002;13(2):214-228

21. Handelman L, Rich M, Bridgemohan CF, Schneider L. Understanding pediatric inner-city asthma: an explanatory model approach. J Asthma. 2004;41(2):167-177.

22. Braganza S, Ozuah PO, Sharif I. The use of complementary therapies in inner-city asthmatic children. J Asthma. 2003;40(7):823-827.

23. Adams SK, Murdock KK, McQuaid EL. Complementary and alternative medication (CAM) use and asthma outcomes in children: an urban perspective. J Asthma. 2007;44(9):775-782

24. Bearison DJ, Minian N, Granowetter L. Medical management of asthma and folk medicine in a Hispanic community. J Pediatr Psychol. 2002;27(4):385-392.

25. Reznik M, Ozuah PO, Franco K, Cohen R, Motlow F. Use of complementary therapy by adolescents with asthma. Arch Pediatr Adolesc Med. 2002;156(10):1042-1044.

26. Pachter LM, Cloutier MM, Bernstein BA. Ethnomedical (folk) remedies for childhood asthma in a mainland Puerto Rican community. Arch Pediatr Adolesc Med. 1995;149(9):982-988.

27. Koinis-Mitchell D, McQuaid EL, Friedman D, et al. Latino caregivers beliefs about asthma: causes, symptoms, and practices. J Asthma. 2008;45(3):205-210.

28. Pachter LM, Weller SC, Baer RD, et al. Variation in asthma beliefs and practices among mainland Puerto Ricans, Mexican-Americans, Mexicans, and Guatemalans. J Asthma. 2002;39(2):119-134.

29. Graham RE, Ahn AC, Davis RB, O'Connor BB, Eisenberg DM, Phillips RS. Use of complementary and alternative medical therapies among racial and ethnic minority adults: results from the 2002 National Health Interview Survey. J Natl Med Assoc. 2005;97(4):535-545.

30. Risser AL, Mazur LJ. Use of folk remedies in a Hispanic population. Arch Pediatr Adolesc Med. 1995;149(9):978-981.

31. Kleinman A. Patients and Healers in the Context of Culture. Berkeley, CA: University of California Press; 1980.

32. Padgett DK. Qualitative Methods in Social Work Research. 2nd ed. Thousand Oaks, CA: Sage Publications, Inc.; 2008.

33. Patton MQ. Qualitative Evaluation and Research Methods. 2nd ed. Beverly Hills, CA: Sage Publications, Inc; 1990.

34. Miller WL, Crabtree BF. Depth interviewing. In: Crabtree BF, Miller WL, eds. Doing Qualitative Research. 2nd ed. Thousand Oaks, CA: Sage Publications, Inc.; 1999:89-107.

35. Qualitative Solutions and Research. NVivo 2.0: Using NVivo in Qualitative Research (Computer Software and Manual). Melbourne, Australia: QSR International; 2002.

36. Borkan J. Immersion/Crystallization. In: Crabtree B, Miller W, eds. Doing Qualitative Research. 2nd ed. Thousand Oaks, CA: Sage Publications; 1999.

37. Kuzel AJ, Like RC. Standards of trustworthiness for qualitative studies in primary care. In: Norton PG, Stewart M, Tudiver F, Bass MJ, Dunn EV, eds. Primary Care Research: Traditional and Innovative Approaches. Vol 1. Newbury Park, CA: Sage Publications; 1991:138-158.

38. Harwood A. The hot-cold theory of disease. Implications for treatment of Puerto Rican patients. JAMA. 1971;216(7):1153-1158.

39. Helman CG. Culture, Health, and IIIness. 5th ed. London, UK: Hodder Arnold; 2007

40. Lincoln Y, Guba EG. Naturalistic Inquiry. Newbury Park, CA: SAGE Publications, Inc; 1985 